\title{
POLARIZATION OF BURSTS OF SOLAR RADIO EMISSION AT MICROWAVE FREQUENCIES
}

\author{
H. TANAKA AND T. KAKINUMA \\ Research Institute of Atmospherics, Nagoya University \\ Toyokawa, Japan
}

The intensity and polarization of solar radio bursts at $9400,3750,2000$, and $1000 \mathrm{Mc} / \mathrm{s}$ have been continuously observed since $1957 \mathrm{July}$. At $3750 \mathrm{Mc} / \mathrm{s}$, the linearly polarized components were also measured during 4 months from 1957 October till 1958 January [1]. The analysis of observations to 1958 May reveals the following points:

1. Many bursts at these frequencies show a difference in the intensity of two circularly polarized components. Frequency distributions of maximum degree of polarization of bursts are shown in Fig. 1, where weak bursts are excluded to reduce errors. At 3750 , 2000 , and $1000 \mathrm{Mc} / \mathrm{s}$, particularly at $3750 \mathrm{Mc} / \mathrm{s}$, the bursts of small degree of polarization are predominant. Circularly polarized bursts are occasionally observed at 2000 and $1000 \mathrm{Mc} / \mathrm{s}$, but at higher frequencies the degree of polarization is usually less than 50 per cent.

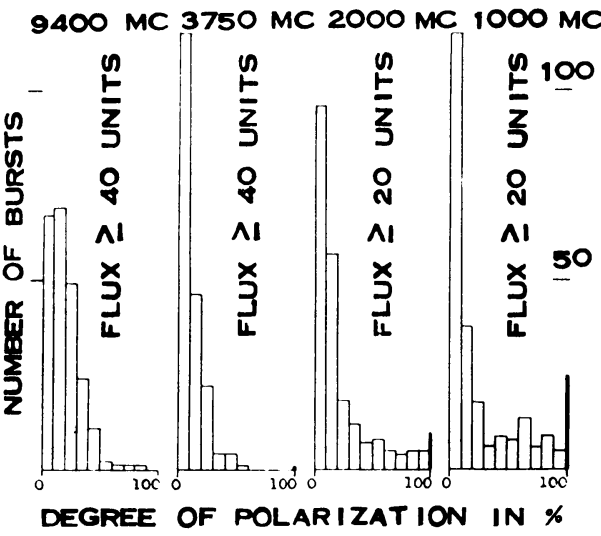

FIG. 1. Histograms showing the frequency distribution of maximum degree of polarization of bursts.

2. At $3750 \mathrm{Mc} / \mathrm{s}$, no linearly polarized components could be detected in 117 bursts for a bandwidth of 10 $\mathrm{Mc} / \mathrm{s}$. The burst at this frequency is usually composed of a small degree of circularly polarized component and a randomly polarized one.

3. Sometimes the sense of polarization varies with frequency. An example is shown in Fig. 2. On this occasion, the degree of polarization is small at $3750 \mathrm{Mc} / \mathrm{s}$ and the sense of polarization reverses between 2000 and $3750 \mathrm{Mc} / \mathrm{s}$. Roughly grouped in Table I are 129 bursts simultaneously observed at four frequencies. The reversal of sense was found in 64 per cent of such bursts. Sometimes the sense of polarization varies with time, as may be found in our monthly report. Some of such phenomena may be interpreted by the motion of the source from the preceding part of a bipolar spot group to the following one or vice versa [4].

4. In Fig. 3, the bursts associated with flares are classified in three types 


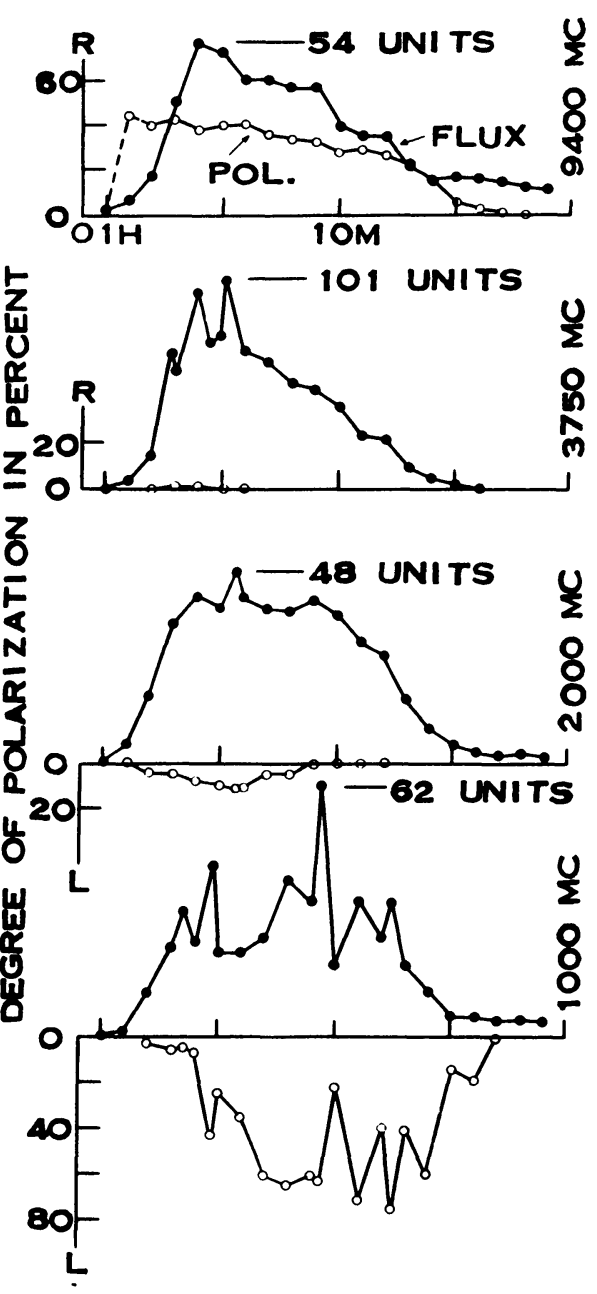

FIG. 2. Intensity and polarization of the burst observed on 1957 July 15.

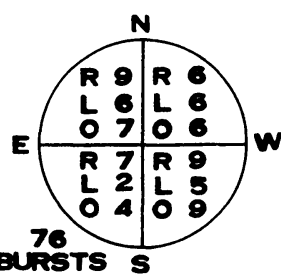

$1000 \mathrm{mc}$

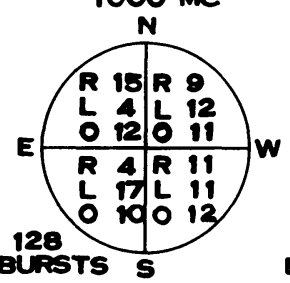

3750 MC
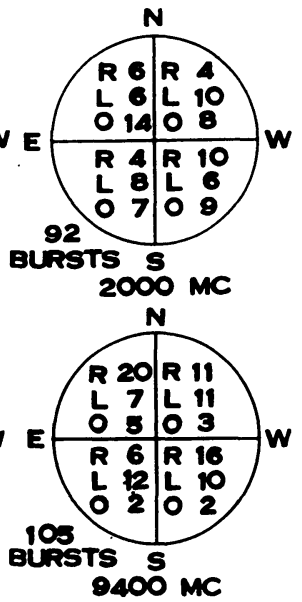

FIG. 3. Relation between position and number of bursts for each polarization.

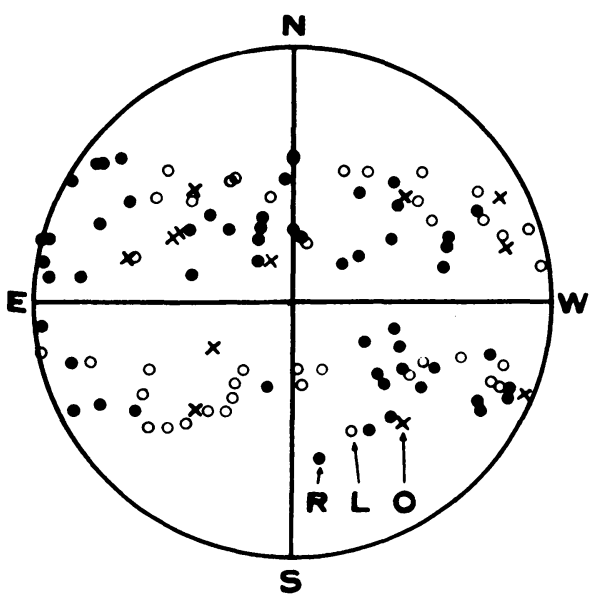

FIG. 4. Relation between position and polarization of bursts at $9400 \mathrm{Mc} / \mathrm{s}$.

TABLE I

\section{Classification of BURSts}

The sense reverses

The sense is the same at all frequencies $\ldots \ldots \ldots \ldots \ldots \ldots \ldots . . .13$

Degree of polarization is nearly zero, etc..................33 33 
as regards the sense of polarization, and they are allocated to each of four quadrants on the solar disk according to the position of the flares. At higher frequencies, the sense of polarization seems to correlate with the position of the source as in the case of $S$ component [2,3]. A precise distribution map at $9400 \mathrm{Mc} / \mathrm{s}$ is shown in Fig. 4.

The results of eclipse observations show that the source of polarization is confined in a small area, about one minute of arc in diameter, over the spot and most sources have the extraordinary sense, as shown in Fig. 5 [4]. Small sources of bursts covering a single sunspot polarity, therefore, are likely to cause random distribution of the sense of polarization over the solar disk. A tendency to the regular distribution at $9400 \mathrm{Mc} / \mathrm{s}$ may be explained by assuming that there are some sources of bursts covering bipolar sunspot groups and that they emit the excess of extraordinary polarization.

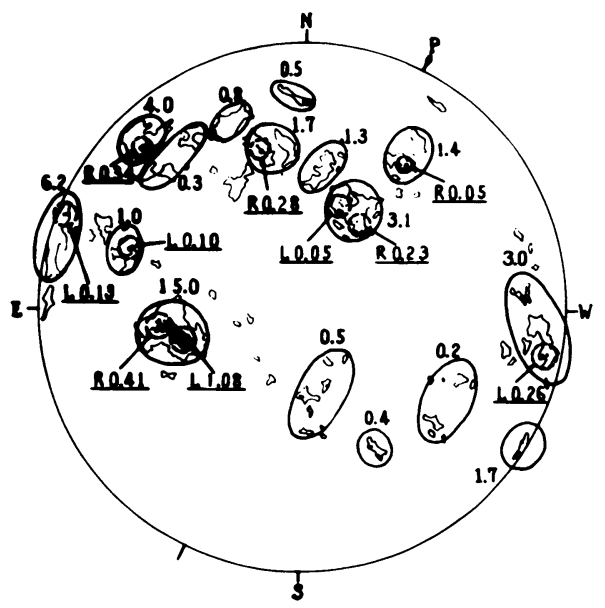

Frg. 5. A $3750-\mathrm{Mc} / \mathrm{s}$ map of $S$ component on 1958 April 19 derived from eclipse observations. Total flux $=100$ per cent $(207$ units), $B$ component $=58.9$ per cent, $S$ component $=41.1$ per cent. Similar maps have been made for 9400,2000 , and $1000 \mathrm{Mc} / \mathrm{s}$.

REFERENCES

[1] Tanaka, H. and Kakinuma, T. Proc. Res. Inst. Atm. Nagoya U. 5, 77, 1958.

[2] Piddington, J. H., and Minnett, H. C. Aust. J. Sci. Res. A. 4, 131, 1951.

[3] Kakinuma, T. Proc. Res. Inst. Atm. Nagoya U. 4, 78, 1956.

[4] Tanaka, H., and Kakinuma, T. Rep. Ionos. Res., Japan, 12, 1958. 